\title{
Bases teóricas da reconexão magnética
}

\author{
Theoretical foundations of magnetic reconnection
}

\author{
V.M. Souza*1, M.V.D. Silveira ${ }^{2}$, D. Koga ${ }^{1}$, P.R. Jauer ${ }^{1}$ \\ ${ }^{1}$ Instituto Nacional de Pesquisas Espaciais - INPE, São José dos Campos, SP, Brasil. \\ ${ }^{2}$ NASA Goddard Space Flight Center, Code 674, Greenbelt, Maryland, USA.
}

Recebido em 11 de agosto de 2015. Aceito em 30 de setembro de 2015

\begin{abstract}
Neste trabalho, o conceito e a origem histórica do processo físico conhecido como reconexão magnética são apresentados, bem como um dos primeiros modelos analíticos que serviu como base teórica para futuras investigações do fenômeno. A reconexão pode ocorrer entre dois ou mais regimes de plasmas magnetizados próximos o suficiente para que efeitos magneto-hidrodinâmicos não ideais permitam a reestruturação topológica dos seus respectivos campos magnéticos. Como consequência, o plasma pode ser acelerado num curto espaço de tempo, tal como observado em fenômenos físicos explosivos como, por exemplo, as explosões (flares) solares. Focam-se nos primeiros esforços analíticos propostos para explicar o processo segundo a perspectiva da magneto-hidrodinâmica. A reconexão magnética tem o potencial de tornar-se um mecanismo universal o qual pode ser utilizado no auxílio ao entendimento de uma miríade de processos físicos ocorrendo tanto em plasmas de laboratório, como em plasmas astrofísicos.
\end{abstract}

Palavras-chave: reconexão magnética, física de plasmas, magneto-hidrodinâmica.

In this work both the concept and historical origins of the physical process known as magnetic reconnection are presented, as well as one of the first analytical models which was used as theoretical basis for future investigations on the phenomenon. Magnetic reconnection can occur between two or more magnetized plasma regimes which are close enough to allow non-ideal magnetohydrodynamic effects to take place and consequently change the topological structure of the interacting magnetic fields. As a result, the plasma can be accelerated in a short amount of time, as has been observed in explosive physical phenomena like solar flares. In this work, it is emphasized the first mathematical efforts employed in order to understand and describe reconnection according to the point of view of magnetohydrodynamics. Magnetic reconnection has the potential of becoming a universal mechanism which can be used to help to understand a myriad of physical processes occurring in both laboratory and astrophysical plasmas.

Keywords: magnetic reconnection, plasma physics, magnetohydrodynamics.

\section{Introdução}

Em praticamente todos os recantos do Universo, encontrar-se-á matéria em forma de plasma que por sua vez possui algum campo magnético intrínseco. Em geral, quando dois ou mais regimes distintos de plasmas encontram-se eles podem interagir de tal forma que suas respectivas configurações magnéticas tornam-se interconectadas, e portanto um "portal" é criado entre eles estabelecendo uma permuta de energia, massa e momento [1]. Como resultado, a energia do sistema, inicialmente armazenada no

*Endereço de correspondência: vitor.souza@inpe.br campo magnético, é convertida em energias cinética e térmica do plasma. Um único mecanismo é capaz de reunir todas essas características: a reconexão magnética 2].

Historicamente, R.G. Giovanelli 3 e F. Hoyle [4] foram os primeiros a sugerir que a reconexão magnética seria capaz de acelerar e aquecer o plasma em eventos de explosões (flares) solares e subtempestades magnéticas [5]. Contudo, foi o aluno de F. Hoyle, J.W. Dungey [6], o primeiro a sugerir que: "linhas de força magnética podem ser quebradas e reconectadas" em uma limitada região na qual intensas correntes elétricas são formadas. Dungey 6] 
também sugere que a reconexão magnética é a precursora do processo de formação das auroras, e pode ser utilizada no auxílio à compreensão do fenômeno de explosões solares.

Seguindo o trabalho pioneiro de Dungey [6], P.A. Sweet [7] e E.N. Parker [8] formularam, independentemente, o primeiro modelo magneto-hidrodinâmico (MHD) estacionário para descrever o processo de reconexão. A taxa de reconexão neste modelo, ou seja, a taxa com que energia magnética é convertida em energias cinética e térmica, depende da resistividade elétrica do plasma e da dimensão espacial do sistema. Foi constatado posteriormente [9] que a taxa de reconexão no modelo de Sweet-Parker era muito lenta (por um fator de $\sim 100$ menor) para explicar fenômenos tais como as explosões solares. Por este fato, o modelo de Sweet-Parker ficou conhecido como modelo de reconexão magnética lenta. Já Petschek [10] desenvolveu um modelo alternativo, ainda MHD, no qual a dimensão espacial do sistema poderia ser arbitrariamente reduzida, em comparação à utilizada no modelo de Sweet-Parker, de tal forma a aumentar a taxa de reconexão para valores capazes de explicar os fenômenos de explosões solares. O modelo de Petschek [10] foi, então, o primeiro modelo de reconexão magnética rápida.

Embora o tópico de explosões (flares) solares fornecesse (e ainda continua a fornecer) um nicho para desenvolvimento da teoria de reconexão magnética, a descoberta da chamada cauda magnética [11], a qual possuia uma configuração de campo magnético favorável à aplicação do conceito de reconexão, bem como o crescente interesse nos estudos e observações de subtempestades magnéticas [12], fez com que o ambiente próximo à Terra, conhecido como magnetosfera terrestre, fosse cada vez mais utilizado para testar o crescente número de modelos de reconexão magnética. O trabalho de Dungey [13] sobre como a configuração topológica do campo magnético terrestre seria modificada a partir da interação, via reconexão magnética, com o vento solar magnetizado, abriu as portas para a pesquisa de reconexão na magnetosfera terrestre. Desde então, diversas investigações tanto teóricas [14 18, quanto com observações in situ de satélites no espaço próximo à Terra [19 30 tem confirmado a presença do processo de reconexão magnética como principal mediador da chamada interação vento solar-magnetosfera terrestre.
Além da magnetosfera terrestre, constatou-se por meio de observações de satélites que a reconexão magnética ocorre, também, em outras magnetosferas planetárias, como a de Saturno [31]; na parte mais externa da atmosfera do Sol: a coroa solar 32, como também em plasmas de laboratório [33]. Estudos computacionais [34 mostram que o mecanismo de reconexão pode ocorrer em coroas de discos de acresção em núcleos ativos de galáxias. Neste caso, Schopper e cols. 34 mostraram que a reconexão magnética é capaz de eficientemente acelerar elétrons a aproximadamente 99,9999\% da velocidade da luz. Percebe-se, portanto, que a reconexão magnética tem potencial para unificar uma vasta gama de fenômenos físicos em um único princípio universal, conquanto todas as respostas concernentes ao processo de reconexão ainda não tenham sido respondidas, como por exemplo, os mecanismos ligados à chamada microfísica ${ }^{1}$ da reconexão, que estão diretamente relacionados com as causas da mudança topológica do campo magnético [35]. Este tópico, no entanto, não será tratado neste artigo.

Este trabalho está organizado da seguinte maneira: na seção 2, conceitos básicos de plasma e teoria MHD de plasma são apresentados no intuito de estabelecer as bases para a descrição (MHD) de reconexão magnética que será apresentada na seção 3. Nesta mesma seção (3), o modelo MHD bidimensional, em estado estacionário, de Sweet-Parker é apresentado. Em seguida, na seção 4 é mostrado um exemplo de evidência observacional do processo de reconexão ocorrendo na natureza. Por fim, as considerações finais são mostradas na seção 5 .

\section{Abordagem MHD da reconexão mag- nética}

Nesta seção, o conceito de plasma é exposto, bem como os critérios estabelecidos para sua existência. Em seguida, o conceito de reconexão é apresentado sob a perspectiva da teoria magneto-hidrodinâmica.

\subsection{Plasma: Conceitos e critérios de exis- tência}

A importância e complexidade do processo de reconexão magnética estão atreladas ao fato do mesmo

\footnotetext{
${ }^{1}$ Fenômenos físicos ocorrendo numa escala espacial da ordem de, ou menor do que, o comprimento inercial do íon, $\lambda_{i}=c / \omega_{p i}$, em que $c$ é a velocidade da luz e $\omega_{p i}$ é a frequência de plasma dos íons.
} 
ocorrer em um meio conhecido como plasma. Necessário é, portanto, que discorramos sobre as características fundamentais deste quarto estado da matéria. Para uma descrição mais completa de plasmas e suas aplicações, recomenda-se a leitura de Bittencourt [36] e Baumjohann e Treumann [37].

De acordo com Bittencourt [36], o plasma é definido como uma substância macroscopicamente neutra contendo elétrons livres e átomos ou moléculas ionizadas. Tais partículas exibem um comportamento coletivo devido à presença de forças de longo alcance, como as forças de Coulomb que variam com o inverso do quadrado da distância. No entanto, a influência de uma única partícula carregada sobre as demais no interior de um plasma é efetiva dentro de uma região de ação bem definida e que, por sua vez, depende das caractéristicas do plasma como temperatura e densidade de partículas eletricamente carregadas. O raio da região de influência é conhecida como comprimento de Debye, $\lambda_{D}$, e é dado por [36, Eq. (2.3)]

$$
\lambda_{D}=\left(\frac{\epsilon_{o} k_{B} T}{n e^{2}}\right)^{1 / 2}
$$

em que $T$ e $n$ são, respectivamente, a temperatura e densidade numérica (número de partículas por unidade de volume) de partículas carregadas, enquanto $\epsilon_{o}, k_{B}$ e $e$ correspondem à permissividade elétrica do vácuo, a constante de Boltzmann e a carga elétrica do elétron, respectivamente. Ilustremos o significado da Eq. (1) considerando a seguinte situação. Considerando uma partícula de carga positiva $q$ no interior de um plasma, o campo elétrico $E_{q}$ associado à esta partícula exercerá influência apenas nas partículas carregadas que estiverem dentro da região de ação definida por uma esfera de Debye ${ }^{2}$ ou seja, a uma distância $r \lesssim \lambda_{D}$. Isto é, o plasma efetivamente blinda, ou limita, o campo de ação de quaisquer campos eletrostáticos gerados por partículas carregadas até uma distância da ordem do comprimento de Debye. É dentro desse limiar de influência $\left(r \lesssim \lambda_{D}\right)$ que uma determinada partícula carregada dentro de um plasma interage coletivamente com as partículas circunvizinhas. Fora desse limiar, $r \gg \lambda_{D}$, é que se diz que, na ausência de distúrbios externos, o plasma é macroscopicamente neutro.

\footnotetext{
${ }^{2}$ Uma esfera dentro do plasma cujo raio é o comprimento de Debye, $\lambda_{D}$.
}

É importante esclarecer que nem todos os meios contendo partículas carregadas são classificados como plasmas, necessitando, para isso, que critérios sejam satisfeitos. O primeiro critério requer que a dimensão característica do plasma em estudo $(L)$ seja muito maior que o comprimento de Debye, ou seja

$$
L \gg \lambda_{D}
$$

Tal condição garante que haja espaço suficiente para que o efeito coletivo de blindagem mencionado acima possa ocorrer. Note que a Eq. (2) já implica em neutralidade macroscópica do plasma, uma vez que se saiba que desvios da neutralidade macroscópica podem ocorrer em escalas de comprimento da ordem do comprimento de Debye. Já o segundo critério expresso pela Eq. (3)

$$
n \lambda_{D}^{3} \gg 1
$$

nos diz que é necessário que o número de partículas dentro da esfera de Debye, aproximadamente dado por $n^{-1 / 3}$, seja muito grande, uma vez que a blindagem eletrostática é um efeito do comportamento coletivo das partículas no interior de uma esfera de Debye.

O terceiro critério para a existência de um plasma é que se o mesmo não for completamente ionizado, ou seja, se houver a presença de partículas neutras, é necessário que a frequência de colisões, $\nu$, das partículas carregadas com as neutras seja menor que a frequência natural de oscilação dos elétrons, $\omega_{p e}$, segundo a qual os movimentos coletivos ocorrem. Logo,

$$
\nu<\omega_{p e}
$$

Se a Eq. (3) não é satisfeita, o plasma não será capaz de comportar-se de uma forma independente sendo, portanto, forçado, via colisões, a entrar em equilíbrio com as partículas neutras, e então o meio pode ser considerado como um gás neutro, perdendo, assim, a característica de plasma.

Em nosso sistema solar, por exemplo, o material que preenche o meio interplanetário (vento solar) encontra-se em estado de plasma. O vento solar é constituído pelo mesmo número de íons e elétrons. Dentre os íons, aproximadamente $95 \%$ correspondem ao Hidrogênio ionizado $\left(\mathrm{H}^{+}\right)$. Já em menores proporções observam-se também moléculas de Hélio duplamente ionizado $\left(\mathrm{He}^{++}\right)$e outros íons mais pesados. A densidade numérica típica do vento solar é 
de 5 partículas por centímetro cúbico $\left(n \sim 5 \mathrm{~cm}^{-3}\right)$, enquanto a temperatura é da ordem de $T \sim 10^{4} \mathrm{~K}$, logo o comprimento de Debye típico para o nosso sistema solar é da ordem de algumas dezenas a centenas de metros. Este valor é muitas ordens de grandeza menor que a dimensão característica do sistema solar, que é da ordem de, pelo menos, uma unidade astronômica $\left(1 \mathrm{UA}^{3}\right)$. Portanto, o primeiro critério (Eq. (2)) para existência do plasma é obedecido no sistema solar, bem como os demais critérios (Eqs. (3) e (44) que podem facilmente ser demonstrados.

\subsection{Teoria MHD do plasma}

Nesta abordagem, trata-se o plasma como um fluido condutor, sem a necessidade de especificar suas várias espécies individuais. Como mencionado acima, o vento solar, por exemplo, é constituído por diversas espécies de íons: $\mathrm{H}^{+}, \mathrm{He}^{++}$, etc. As propriedades macroscópicas do plasma são então determinadas somando-se as contribuições dos diversos tipos de íons presentes. As densidades de massa, $\rho_{m}$, e de carga elétrica, $\rho$, por unidade de volume são obtidas da seguinte maneira 36

$$
\begin{gathered}
\rho_{m}=\sum_{\alpha} \rho_{m \alpha}=\sum_{\alpha} n_{\alpha} m_{\alpha}, \\
\rho=\sum_{\alpha} n_{\alpha} q_{\alpha},
\end{gathered}
$$

em que $\alpha$ denota as diferentes espécies de íons e elétrons presentes no plasma, $m_{\alpha}$ suas respectivas massas e $n_{\alpha}$ suas densidades de carga por unidade de volume. Já a velocidade média do fluido como um todo, v, é dada por

$$
\mathbf{v}=\frac{\sum_{\alpha} \rho_{m \alpha} \mathbf{v}_{\alpha}}{\rho_{m}},
$$

logo, v é definido como uma média ponderada na qual a velocidade de cada espécie tem um peso proporcional à sua densidade de massa. De forma análoga, pode-se definir os outros parâmetros macroscópicos do plasma, tais como a densidade de corrente elétrica, J, e o tensor pressão $\mathcal{P}$. Deste modo, pode-se escrever as equações da magnetohidrodinâmica (MHD) que acoplam as equações que traduzem leis de conservação de massa, momento e energia, com as equações de Maxwell que

\footnotetext{
${ }^{3} 1$ UA, Unidade Astronômica, corresponde à distância média da Terra ao Sol de, aproximadamente, 150.000.000 de quilômetros.
}

regem os campos elétricos e magnéticos presentes no plasma. De particular interesse para nós, é a chamada equação de indução

$$
\frac{\partial \mathbf{B}}{\partial t}=-\nabla \times(-\mathbf{v} \times \mathbf{B}+\mathbf{R}),
$$

que rege a evolução temporal do campo magnético no plasma. A Eq. 8 é obtida substituindo na equação de Faraday-Lenz $(\partial \mathbf{B} / \partial t=-\nabla \times \mathbf{E})$, o campo elétrico, $\mathbf{E}$, da lei de $\mathrm{Ohm}$ generalizada

$$
\mathbf{E}=-\mathbf{v} \times \mathbf{B}+\mathbf{R},
$$

em que $\mathbf{R}$ é dado por

$$
\mathbf{R}=\eta \mathbf{J}+\frac{\mathbf{J} \times \mathbf{B}}{n e}-\frac{\nabla \cdot \mathcal{P}_{e}}{n e}+\frac{m_{e}}{n e^{2}} \frac{\partial \mathbf{J}}{\partial t} .
$$

O termo $\mathbf{R}$ presente nas Eqs. (8) e (9) representa contribuições para o campo elétrico presente no plasma devido aos efeitos resistivo $(\eta \mathbf{J})$, ou seja, que leva em conta as colisões entre elétrons e íons no plasma; da força de Lorentz $(\mathbf{J} \times \mathbf{B})$, também conhecida como termo Hall; efeitos térmicos representados pela divergência do tensor pressão anisotrópica dos elétrons $\left(\nabla \cdot \mathcal{P}_{e}\right)$; e efeitos devidos à contribuição da inércia dos elétrons ao fluxo de corrente elétrica $(\partial \mathbf{J} / \partial t)$. A depender da situação estudada, alguns destes termos podem ser negligenciados. No caso que iremos abordar aqui, somente o termo resistivo $(\eta \mathbf{J})$ é de interesse, uma vez que o modelo simples de reconexão magnética que será apresentado mais adiante analisa a influência deste termo no processo. Contudo, os demais termos são de fundamental importância para o estudo da reconexão magnética quando procura-se entender a microfísica do fenômeno 4

\section{3. "Congelamento" e difusão do campo magnético no plasma}

No caso particular quando $\mathbf{R} \approx \overrightarrow{0}$, obtém-se o modelo MHD ideal. Neste modelo, considera-se que o fluido é um condutor perfeito (resistividade, $\eta$, nula) e a Eq. (8) reduz-se a uma equação de convecção $(\partial \mathbf{B} / \partial t=\nabla \times(\mathbf{v} \times \mathbf{B}))$. Sob estas condições, podese mostrar [38] que o plasma está "congelado" ao campo magnético, isto é, movimentos das partículas

${ }^{4} \mathrm{E}$ com este intuito que a missão Magnetospheric Multi Scale/MMS 35] da NASA foi elaborada, e dentro em breve estará fornecendo dados observacionais que irão auxiliar na resposta a questões ainda abertas nesta área de pesquisa. 
carregadas perpendicularmente à direção do campo magnético local podem ocorrer se, e somente se, a linha de campo magnético, em torno da qual as partículas giram entorno, desloca-se com o plasma nesta direção. Um corolário desta afirmação é que elementos de plasma conectados à uma linha de campo em um determinado instante de tempo, permanecerão conectados à mesma linha de campo em instantes subsequentes. As partículas de plasma não encontram, no entanto, nenhuma restrição de movimento ao longo da linha de campo. Devido à alta condutividade do plasma, condição esta que é satisfeita na maioria dos plasmas que se tem conhecimento no universo, o campo magnético não consegue difundir-se eficientemente no plasma. Se o tamanho do sistema em estudo $(L)$ for também muito grande, o que é o caso dos plasmas cósmicos, o tempo característico de difusão do campo magnético no plasma, $\tau_{D} \sqrt[5]{5}$ pode ser da ordem de dias, ou em casos mais extremos, da ordem da idade do Universo!

Considerando o plasma magnetizado do vento solar, por exemplo, durante todo o trajeto $\left(\sim 1,5 \times 10^{11} \mathrm{~m}\right)$ que ele leva para atingir a Terra, ou seja, cerca de 3,5 dias em condições típicas ${ }^{6}$ o campo magnético difunde-se neste plasma ao longo de uma distância $L$ da ordem de $10^{3}$ metros apenas. Isto é, o campo magnético no vento solar está praticamente congelado e é transportado juntamente com o plasma [37.

Em regime de MHD ideal, reconexão magnética não ocorre. É necessário, portanto, que algum fenômeno quebre a condição de congelamento e permita que o campo magnético desacople-se do plasma e possa difundir-se pelo mesmo, permitindo que a reconexão magnética se estabeleça. Tal ocasião ocorre exatamente quando o termo $\mathbf{R}$ na Eq. (10) é diferente de zero e comparável ao termo $-\mathbf{v} \times \mathbf{B}$. Isto ocorre em regiões delimitadas do espaço nas quais os campos magnéticos dos plasmas interagentes aniquilam-se, dando origem a intensas correntes elétricas. Tais regiões são conhecidas como região de difusão, e é nelas que os campos magnéticos tem

\footnotetext{
${ }^{5} \tau_{D}=\mu_{o} L^{2} / \eta$ é o tempo característico que o campo magnético leva para difundir-se num plasma de condutividade $\sigma_{o}=1 / \eta$, escala de comprimento $L$ e permeabilidade magnética do vácuo $\mu_{o}$. Para o núcleo da Terra, por exemplo, considerando que o mesmo seja constituído de ferro fundido, $\tau_{D}$ é da ordem de $10^{4}$ anos, já para o campo magnético do Sol, $\tau_{D}$ é da ordem de $10^{10}$ anos!

${ }^{6}$ Velocidade do vento solar $\sim 500 \mathrm{~km} / \mathrm{s}$.
}

suas configurações topológicas ou, alternativamente, suas conectividades, alteradas.

\subsection{Descrição qualitativa da reconexão mag- nética}

A Fig. 1 mostra um esquema, em duas dimensões, de como a reconexão magnética ocorre para dois regimes de plasmas com campos magnéticos de orientação oposta (setas pretas preenchidas). Em um instante $t<0$, os campos aproximam-se da região central juntamente com o plasma, cuja direção de movimento é representada pelas setas brancas. Neste caso, plasma e campo magnético estão congelados, tal como discutido na seção 2.3 e o único campo elétrico existente é o de convecção dado por $-\mathbf{v} \times \mathbf{B}$, ou seja, $\mathbf{R} \approx \overrightarrow{0}$. Já no instante $t=0$ (painel do meio) a reconexão ocorre $(\mathbf{R} \neq 0)$ entre as linhas de campo magnético que encontram-se na parte central. Estas duas linhas de campo que se tocam forma o que se chama de separatriz, a qual separa regiões de campos magnéticos de diferentes conectividades. Nesta região, os campos paralelos de orientação oposta (antiparalela) aniquilam-se e pela lei de Ampère $\left(\mathbf{J}=\nabla \times \mathbf{B} / \mu_{o}\right)$ uma lâmina de corrente elétrica entrando no plano da página deve ser formada entre os dois regimes de plasma (retângulo cinza na Fig. 1). Nesta lâmina de corrente elétrica o campo elétrico não é mais o de convecção e assume a forma de um (ou mais) termo(s) presente(s) na Eq. 110. A existência deste(s) termo(s) é que permite que a condição de congelamento seja violada e a reconexão magnética se estabeleça.

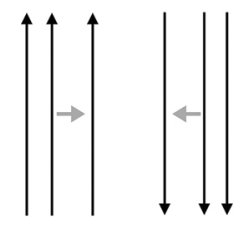

$t<0$

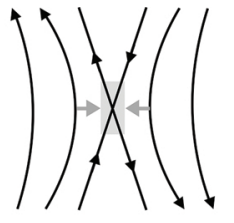

$\mathrm{t}=0$

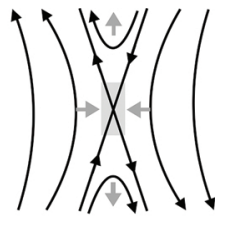

$t>0$
Figura 1: Esquema para ocorrência, em duas dimensões, de reconexão magnética entre campos magnéticos com orientações antiparalelas (setas pretas). Em $t<0$ não há reconexão. $O$ processo inicia-se em $t=0$ e continua para instantes posteriores $(t>0)$. Os plasmas, cuja direção de movimento é representado pelas setas cinzas, encontram-se na região central (em $t=0)$ e em decorrência da reconexão das linhas de campo são expelidos $(t>0)$ desta região (setas cinzas verticais) juntamente com as linhas de campo reconectadas. 
Uma importante ressalva sobre o esquema mostrado na Fig. 1, em particular nos instantes $t=0$ e $t>0$ é o fato de duas linhas de força magnéticas se cruzarem 7 Conquanto imprecisa, o intuito desta representação é mostrar que os campos magnéticos irão reconectar-se neste ponto, ou ainda em outros pontos dentro da lâmina de corrente, embora ainda não se saiba exatamente como este processo ocorre. Sabe-se, entretanto, que a reconexão magnética odebece as leis de Maxwell em sua totalidade, e esforços teóricos, computacionais e observacionais [35] estão sendo empreendidos com o objetivo de compreender todas as particularidades do processo.

Vale ressaltar que o esquema mostrado na Fig. 1 simplifica sobremaneira o processo de reconexão que inerentemente ocorre em uma geometria tridimensional e, em geral, em estado não estacionário. $\mathrm{O}$ objetivo em apresentar tal esquema é que este facilita o entendimento de como (acredita-se que) o mecanismo ocorra para campos magnéticos de orientações precisamente opostas (antiparalelas), bem como o tratamento matemático utilizado para quantificar o processo. Enfatiza-se que quando os campos magnéticos não possuem orientações precisamente antiparalelas, ou seja, quando o ângulo formado entre suas direções difere de $180^{\circ}$, a reconexão magnética ainda pode ocorrer entre as componentes dos campos magnéticos interagentes que possuem uma orientação antiparalela, tal como proposto originalmente por Sonnerup [14] e Gonzalez e Mozer [15]. Este modo de reconexão conhecido como reconexão por componente tem sido observado manifestar-se tanto na magnetosfera terrestre 19,20 , como em plasmas de laboratório [33. Conquanto importante, os detalhes deste processo não serão abordados neste trabalho, uma vez que se tratam de derivações dos modelos de reconexão iniciais, e aqui pretende-se apenas apresentar os primeiros esforços empreendidos na tentativa de compreender o fenômeno.

\section{Descrição quantitativa da reconexão magnética}

Nesta seção é apresentado o modelo pioneiro de Sweet-Parker [7], que traduziu um dos primeiros esforços de quantificar o processo de reconexão

\footnotetext{
${ }^{7} \mathrm{O}$ conceito de linhas de força, proposto por Michael Faraday, é um artifício matemático utilizado para auxiliar na visualização de campos vetoriais, tais como os campos elétrico e magnético.
}

magnética, em particular a chamada taxa de reconexão.

\subsection{Modelo para reconexão magnética bi- dimensional em estado estacionário: Sweet-Parker}

O modelo de Sweet-Parker utiliza a abordagem MHD para descrever a reconexão magnética, em estado estacionário, devido ao efeito de uma resistividade finita $\eta$. Neste caso, o campo elétrico no referencial do laboratório é dado por:

$$
\mathbf{E}=-\mathbf{v} \times \mathbf{B}+\eta \mathbf{J} .
$$

Esta resistividade é gerada por colisões coulombianas entre elétrons e íons pertencentes a plasmas com campos magnéticos antiparalelos que se aproximam um do outro com velocidades não relativísticas. Tal resistividade é importante somente no interior da lâmina de corrente de comprimento $2 L_{s}$ e espessura $2 \delta$ que separa os plasmas interagentes e na qual o campo magnético reverte sua orientação (ver Fig. 2]. Fora da lâmina de corrente, no entanto, o plasma está congelado ao campo magnético, tal como discutido na seção 2.3 . Todo o processo está em estado estacionário, portanto variações temporais são desprezadas, ou seja, $\partial / \partial t \equiv 0$. Pretende-se, aqui, determinar a taxa de reconexão magnética derivada pelo modelo de Sweet-Parker, a qual pode ser estabelecida através da razão entre a velocidade do plasma que entra na região de reconexão, $V_{i n}$, pela velocidade de saída do plasma desta região,

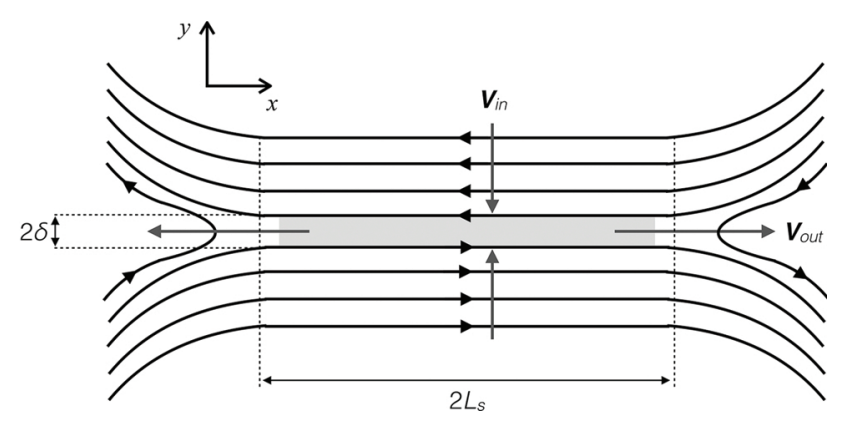

Figura 2: Geometria do modelo de reconexão magnética em estado estacionário de Sweet-Parker. Plasmas com campos magnéticos de orientações opostas (linhas pretas) aproximam-se da lâmina de corrente (área retangular cinza) por cima e por baixo com velocidade $V_{\text {in }}$. O plasma que sai (setas horizontais) da lâmina de corrente é acelerado como consequência do processo de reconexão e atinge uma velocidade $V_{\text {out }}$. 
$V_{\text {out }}$. Em geral, $V_{\text {out }}$ é maior que $V_{\text {in }}$, portanto esta razão fornece uma medida de quanto o plasma foi acelerado. Este acréscimo em velocidade está intimamente relacionado com a conversão de energia magnética em energias cinética e térmica do plasma proporcionada pela reconexão magnética.

A geometria do modelo de reconexão magnética de Sweet-Parker é mostrada na Fig. 2. Os plasmas com mesma densidade de massa $\rho_{m}$ aproximam-se da região central nas direções $\pm y$ com a mesma velocidade de entrada $V_{i n}$. Eles possuem campos magnéticos de mesma intensidade $B$, mas de orientações opostas $( \pm x)$. Como mencionado na seção 2.4. uma lâmina de corrente deve ser estabelecida na região de contato dos plasmas separando estes dois regimes. Pela configuração de campos magnéticos mostrada na Fig. 2, a corrente elétrica, consistente com a lei de Ampère, possui uma direção saindo do plano da página (direção $+z$ ). Nesta região, de acordo com o modelo, o campo elétrico é considerado ser inteiramente especificado pelo termo resistivo $\eta J_{z} \equiv \eta J$, isto é, $\left.E \sim \eta J\right]^{8}$ Já fora desta região, o campo elétrico é puramente convectivo, $E \sim V_{y} B_{x} \equiv V_{i n} B$, e também aponta para fora do plano da página. O fato do campo elétrico ser o mesmo tanto fora como dentro da região de lâmina de corrente é que, em estado estacionário, a lei de Faraday estabelece que o rotacional do campo elétrico deve ser nulo $(\nabla \times \mathbf{E}=\overrightarrow{0})$, o que implica que o campo elétrico não deve possuir nenhuma descontinuidade ou, alternativamente, que ele deve ser espacialmente uniforme $\left(\eta J \sim V_{i n} B\right)$. A corrente elétrica, por sua vez, pode ser determinada em função da meia espessura $\delta$ da lâmina de corrente, e como consequência é possível expressar a velocidade de entrada do plasma em função dos parâmetros $\delta$ e $\eta$

$$
J=\frac{|\nabla \times \mathbf{B}|}{\mu_{o}} \sim \frac{B}{\mu_{o} \delta} \sim \frac{V_{i n} B}{\eta} \longrightarrow V_{i n} \sim \frac{\eta}{\mu_{o} \delta} .
$$

Agora, necessita-se determinar a velocidade de saída do plasma da região de difusão. Imediatamente após a reconexão magnética, as linhas de campo reconectadas estão bastante torcidas, tais como as linhas interceptadas pelas setas horizontais mostradas na extrema esquerda e direita da Fig. 2. Tais linhas tendem a relaxar para um estado de menor energia, no qual as linhas retorcidas tendem a tornar-se retas. À medida que relaxam, estas linhas convertem

$\overline{{ }^{8} \mathrm{O} \text { símbolo " } " \text { significa }}$ "da ordem de". energia magnética em energia cinética do plasma, acelerando as partículas que vão movendo-se com estas linhas de campo e atingindo uma velocidade $V_{\text {out }}$. Relembrando que a massa no sistema em estudo deve ser conservada, ou seja, a quantidade de plasma que entra na lâmina de corrente deve ser igual à que sai, e que neste modelo efeitos de compressibilidade do plasma são desprezados $(\nabla \cdot \mathbf{v}=0)$, pode-se mostrar que

$$
V_{\text {in }} L_{s} \sim V_{\text {out }} \delta .
$$

A lei de Gauss $(\nabla \cdot \mathbf{B}=0)$ estabelece que os campos magnéticos de entrada, $B_{x}$, e saída, $B_{y}$, da região de difusão, relacionam-se da seguinte forma

$$
B_{y} \sim \frac{\delta}{L_{s}} B_{x} .
$$

Utilizando as Eqs. (13) e (14), a velocidade de saída do plasma é obtida equacionando a componente na direção $x$ do termo convectivo de velocidade $((\mathbf{v} \cdot \nabla) \mathbf{v})$ e do termo de curvatura magnética $((\mathbf{B} \cdot \nabla) \mathbf{B})$ presentes na lei de conservação de momento para o caso em estudo

$$
\begin{aligned}
\rho_{m}(\mathbf{v} \cdot \nabla) \mathbf{v} & = \\
\mu_{o}^{-1}(\mathbf{B} \cdot \nabla) \mathbf{B} & \longrightarrow \rho_{m} \frac{V_{\text {out }}^{2}}{L_{s}} \sim \frac{B_{x}^{2}}{\mu_{o} L_{s}},
\end{aligned}
$$

de onde se depreende que a velocidade de saída do plasma é da ordem da velocidade de Alfvén, $V_{A}$, dada por

$$
V_{\text {out }} \sim \frac{B_{x}}{\sqrt{\rho_{m} \mu_{o}}}=V_{A},
$$

que é uma velocidade característica de um plasma magnetizado. Nota-se que a velocidade de saída, $V_{\text {out }}$, do plasma da região de difusão onde o campo magnético sofre a reconexão, isto é, a lâmina de corrente, depende inteiramente dos parâmetros de entrada.

Com o objetivo de estabelecer a taxa de reconexão magnética no modelo de Sweet-Parker, podese fazer a razão $V_{\text {in }} / V_{\text {out }}$, que expressa, como já mencionado acima, a taxa de conversão de energia magnética em energias cinética e térmica do plasma. Utilizando a Eq. (13), juntamente com as Eqs. (12) e (16), encontra-se

$$
\frac{V_{\text {in }}}{V_{\text {out }}} \sim \sqrt{\frac{\eta}{\mu_{o} V_{A} L_{s}}}=S^{-1 / 2}
$$


em que $S=\mu_{o} V_{A} L_{s} / \eta$ é conhecido como número de Lundquist 9 Estudos mostraram que $L_{s}$ no modelo de Sweet-Parker é da ordem da dimensão espacial do sistema $L_{s i s}$, que para plasmas espaciais é um valor muito grande 10 Aplicando o modelo no contexto de explosões solares, pode-se encontrar que a taxa de reconexão é da ordem de $\sim 2 \times 10^{-7}$ [39], o que dá uma velocidade de entrada $V_{\text {in }}$ de somente $\sim 0,8 \mathrm{~m} / \mathrm{s}$. Logo, o tempo caraterístico para que a energia magnética seja liberada é da ordem de $L_{\text {sis }} / V_{\text {in }} \sim 10^{7} \mathrm{~s}$, que é em torno de 4 ordens de magnitude maior do que o observado (algo em torno de $10^{3} \mathrm{~s}$ ) para fenômenos de explosões solares. Além disso, o processo colisional adotado por Sweet-Parker para promover a reconexão magnética na região de difusão não é muito efetivo, uma vez que plasmas espaciais são praticamente não colisionais, e a taxa de reconexão no modelo varia com $\eta^{1 / 2}$. Por estes motivos, o modelo de Sweet-Parker é considerado como modelo de reconexão magnética lenta.

Em plasmas nos quais as colisões entre os seus constituintes tem um papel importante, tais como plasmas de laboratório, o modelo de Sweet-Parker reproduz os resultados experimentais satisfatoriamente. Portanto, o modelo é fisicamente viável, conquanto necessite ser aprimorado para que possa explicar a rápida liberação de energia magnética observada em alguns fenômenos, tais como as explosões solares 39 .

\section{Evidência observacional da reconexão magnética}

A literatura acerca das evidências observacionais do processo de reconexão magnética é vasta (ver seção 1). Pretende-se mostrar aqui um exemplo de como o mecanismo funciona em um sistema real, e como as principais características do fenômeno são observadas e verificadas.

É impossível observar o processo de reconexão magnética a "olhos nus", uma vez que a linha de

\footnotetext{
${ }^{9} \mathrm{O}$ número de Lundquist é um parâmetro adimensional que representa a razão entre o tempo de difusão no plasma devido a efeitos resistivos, $\mu_{o} L^{2} / \eta$, e o tempo para uma onda de Alfvén propagar-se num plasma com dimensão característica $L$, ou seja, $L / V_{A}$, e $V_{A}$ é a velocidade de Alfvén dada pela Eq. (16). Grandes valores do número de Lundquist significa plasmas com alta condutividade, nos quais os tempos de difusão são muito longos, enquanto pequenos valores indicam um plasma mais resistivo.

${ }^{10}$ Algo da ordem de, ou maior do que, $10^{6}$ metros.
}

força magnética não existe no mundo real! Entretanto, o Sol é um laboratório único na natureza para que possamos literalmente "enxergar" como a reconexão magnética ocorre, dado que plasmas quentes (e brilhantes) nesta estrela deslocam-se transversalmente ou paralelamente às linhas de campo magnético. Para este fim, a utilização de técnicas de imageamento em múltiplos comprimentos de onda [40] tornam-se uma ferramenta apropriada.

A Fig. 3 mostra uma evidência de reconexão magnética tipo Sweet-Parker observada pelo satélite Observatório Dinâmico Solar (SDO, [42]). O SDO observa continuamente o Sol e nos fornece um punhado de informações para entender a dinâmica atividade solar, bem como melhorar a previsão do chamado Clima Espacial, e.g., EMBRACE/INPE ${ }^{11}$ O painel (b) da Fig. 3 mostra uma configuração esquemática do campo magnético coronal inferida a partir das estruturas de plasma aquecidos à aproximadamente 10 milhões de graus Kelvin mostradas no painel (a). A linha vermelha indica a lâmina de corrente, a linha curva tracejada o limbd ${ }^{12}$ solar, e as setas cinza representam as linhas de força. Nesta figura, as linhas de campo magnético lentamente aproximam-se (setas vermelhas) da lâmina de corrente, sendo então reconectadas e ejetadas desta lâmina a altíssimas velocidades (setas azul turquesa). Tal movimento de entrada e saída de plasma da região de reconexão foi observado pelo SDO e a taxa de reconexão estimada variou de $\sim 0,05$ a $0,5[32$. Embora neste exemplo se verifique uma geometria de reconexão consistente com a proposta por Sweet-Parker, notamos que o modelo de Sweet-Parker não seria capaz de explicar o rápido processo de reconexão que ocorre nestes casos. É necessário, portanto, que outros modelos de reconexão sejam incorporados [43].

\section{Considerações finais}

Neste trabalho, mostrou-se a importância do estudo e compreensão da reconexão magnética, cujas características podem ser utilizadas no auxílio ao entendimento de uma miríade de processos físicos ocorrendo tanto em plasmas de laboratório, como em plasmas astrofísicos.

Uma introdução às bases teóricas da reconexão magnética segundo a perspectiva da teoria magnetohidrodinâmica (MHD) foi apresentada. Nesta abor-

\footnotetext{
${ }^{11}$ http://www2 . inpe.br/climaespacial/portal/pt/

${ }^{12}$ Borda do disco solar.
} 

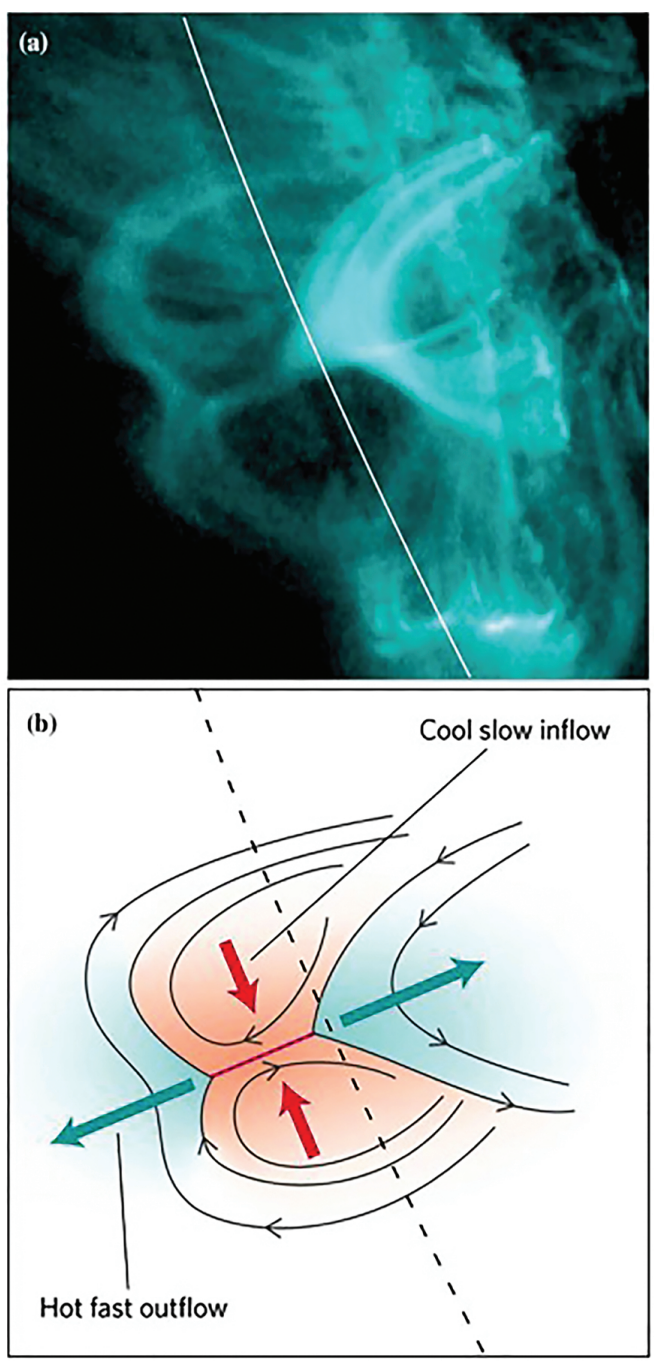

Figura 3: Descrição da Figura 1 de Forbes 41]: (a) uma imagem da coroa solar obtida do instrumento AIA abordo do SDO retirada no comprimento de onda de $131 \AA$, mostrando estruturas de plasma com temperaturas superiores a 10 milhões de graus Kelvin durante uma explosão (flare) solar em 17 de Agosto de 2011. A linha branca curva indica o limbo da face visível do Sol. (b) Esquema mostrando a estrutura do campo magnético e fluxos de plasma inferidos a partir de (a). O segmento de linha em vermelho representa a lâmina de corrente, e a curva tracejada indica o limbo solar. As linhas de campo magnético (linhas cinza) entram lentamente (setas vermelhas) na região da lâmina de corrente, onde reconectam-se. Uma vez reconectadas, elas deixam a região de reconexão em altas velocidades (setas azul turquesa).

dagem, o plasma é tratado como um fluido condutor que obedece às leis de conservação de massa, energia e momento, bem como às equações de Maxwell. Enfatizou-se que a reconexão magnética não ocorre espontaneamente entre plasmas magnetizados que satisfaçam as condições da teoria MHD ideal, na qual o plasma não suporta campos elétricos em seu próprio sistema de referência por possuir uma condutividade extremamente alta. Mostrou-se que são necessários que campos elétricos associados a efeitos não-ideais, tais como efeitos resistivo e Hall, sejam excitados para que a alteração topológica dos campos magnéticos interagentes possa ocorrer, permitindo uma troca de massa, energia e momento entre os distintos regimes de plasma.

Um dos primeiros modelos analíticos, dentro da abordagem MHD, o de Sweet-Parker, foi introduzido, mostrando como a reconexão magnética deveria ocorrer em estado estacionário para uma configuração bidimensional. Um exemplo observacional de reconexão magnética ocorrendo de acordo com a geometria proposta por Sweet-Parker foi apresentado. Embora simples, este modelo serviu de base para o entendimento da Física da reconexão magnética, bem como inspirou a crescente investigação nos aspectos mais fundamentais e ainda não inteiramente compreendidos do processo que, atualmente, são capazes de serem analisados, em sua grande maioria, somente através de simulações computacionais [44].

\section{Agradecimentos}

V.M. Souza agradece à FAPESP, processo número 2014/21229-9, pelo auxílio financeiro e apoio à pesquisa. M.V.D. Silveira e P.R. Jauer agradecem à CNPq, processos número 232906/2014-9 e 505759/2013-6, pelo suporte financeiro. D. Koga agradece ao Programa de Pós-Doutorado da CAPES pelo auxílio financeiro.

\section{Referências}

[1] V.M. Souza, Location of Large Scale Reconnection at Earth's Dayside Magnetopause as Probed by Analytical X-line Models and In Situ Observations. Tese de Doutorado, Instituto Nacional de Pesquisas Espaciais, 2015.

[2] V.M. Vasyliunas, Reviews of Geophysics 13, 303 (1975).

[3] R.G. Giovanelli, Mon. Notices Roy. Astron. Soc. 107, 338 (1947).

[4] F. Hoyle, Some Recent Researches in Solar Physics (Cambridge University Press, Cambridge, 1949), $1^{\text {st }}$ ed.

[5] E. Costa Jr., F.J.R. Simões Jr., F.R. Cardoso e M.V. Alves, Revista Brasileira de Ensino de Física 33, 4301 (2011). 
[6] J.W. Dungey, Phil. Mag. Ser. 7 44, 725 (1953).

[7] P.A. Sweet, in: Electromagnetic Phenomena in Cosmical Physics, editado por B. Lehnert (Cambridge University Press, London, 1958).

[8] E.N. Parker, Journal of Geophysical Research: Space Physics 62, 509 (1957).

[9] E.N. Parker, Astrophys. J., Suppl. 8, 177 (1963).

[10] H.E. Petschek, in: AAS-NASA Symposium on the Physics of Solar Flares, Greenbelt, 1964, editado por Wilmot N. Hess, p. 425.

[11] N.F. Ness, Reviews of Geophysics 7, 97 (1969).

[12] C. Russell and R.McPherron, Space Science Reviews 15, 205 (1973).

[13] J.W. Dungey, Phys. Rev. Lett. 6, 47 (1961).

[14] B.U.Ö. Sonnerup, Journal of Geophysical Research: Space Physics 79, 1546 (1974).

[15] W.D. Gonzalez and F.S. Mozer, Journal of Geophysical Research: Space Physics 79, 4186 (1974).

[16] S.W.H. Cowley, Reviews of Geophysics and Space Physics 20, 531 (1982).

[17] B.T. Tsurutani and W.D. Gonzalez, Geophysical Research Letters 22, 663 (1995).

[18] P.A. Cassak and M.A. Shay, Physics of Plasmas 14, 102114 (2007).

[19] G. Paschmann, B.U.Ö. Sonnerup, I. Papamastorakis, N. Sckopke, G. Haerendel, S.J. Bame, J.R. Asbridge, J.T. Gosling, C.T. Russell and R.C. Elphic, Nature 282, 243 (1979).

[20] B.U.Ö. Sonnerup, G. Paschmann, I. Papamastorakis, N. Sckopke, G. Haerendel, S.J. Bame, J.R. Asbridge, J.T. Gosling and C.T. Russell, Journal of Geophysical Research: Space Physics 86, 10049 (1981).

[21] V. Angelopoulos, T.D. Phan, D.E. Larson, F.S. Mozer, R.P. Lin, K.Tsuruda, H. Hayakawa, T. Mukai, S. Kokubun, T. Yamamoto, D.J. Williams, R.W. McEntire, R.P. Lepping, G.K. Parks, M. Brittnacher, G. Germany, J. Spann, H.J. Singer and K. Yumoto, Geophysical Research Letters 24, 2271 (1997).

[22] T.D. Phan, L.M. Kistler, B. Klecker, G. Haerendel, G. Paschmann, B.U.Ö. Sonnerup, W. Baumjohann, M.B. Bavassano-Cattaneo, C.W. Carlson, A.M. DiLellis, K.H. Fornacon, L.A. Frank, M. Fujimoto, E. Georgescu, S. Kokubun, E. Moebius, T. Mukai, M. Oieroset, W.R. Paterson and H.Reme, Nature 404, 848 (2000).

[23] T. Nagai, I. Shinohara, M. Fujimoto, M. Hoshino, Y. Saito, S. Machida and T. Mukai, Journal of Geophysical Research: Space Physics 106, 25929 (2001).

[24] T.D. Phan, M. Freeman, L. Kistler, B. Klecker, G. Haerendel, G. Paschmann, B.U.Ö. Sonnerup, W. Baumjohann, M. Bavassano-Cattaneo, C. Carlson, A. DiLellis, K.-H. Fornacon, L. Frank, M. Fujimoto, E. Georgescu, S. Kokubun, E. Moebius, T. Mukai, W. Paterson and H. Reme, Earth, Planets and Space 53, 619 (2001).
[25] T.D. Phan, M.W. Dunlop, G. Paschmann, B. Klecker, J.M. Bosqued, H. Rème, A. Balogh, C. Twitty, F.S. Mozer, C.W. Carlson, C. Mouikis and L.M. Kistler, Annales Geophysicae 22, 2355 (2004).

[26] T.D. Phan, H. Hasegawa, M. Fujimoto, M. Oieroset, T. Mukai, R.P. Lin and W. Paterson, Geophysical Research Letters 33, (2006).

[27] V. Angelopoulos, J.P. McFadden, D. Larson, C.W. Carlson, S.B. Mende, H. Frey, T.D. Phan, D.G. Sibeck, K.-H. Glassmeier, U. Auster, E. Donovan, I.R. Mann, I.J. Rae, C.T. Russell, A. Runov, X.-Z. Zhou and L. Kepko, Science 321, 931 (2008).

[28] M.W. Dunlop, Q.-H. Zhang, Y.V. Bogdanova, M. Lockwood, Z. Pu, H. Hasegawa, J. Wang, M.G.G.T. Taylor, J. Berchem, B. Lavraud, J. Eastwood, M. Volwerk, C. Shen, J.-K. Shi, D. Constantinescu, H. Frey, A.N. Fazakerley, D.G. Sibeck, P. Escoubet, J.A. Wild and Z.-X. Liu, Physical Review Letters 107, 25004 (2011).

[29] T.D. Phan, G. Paschmann, J.T. Gosling, M. Oieroset, M. Fujimoto, J.F. Drake and V. Angelopoulos, Geophysical Research Letters 40, 11 (2013).

[30] B.M. Walsh, T.D. Phan, D.G. Sibeck and V.M. Souza, Geophysical Research Letters 41, 223 (2014).

[31] S.A. Fuselier, R. Frahm, W.S. Lewis, A. Masters, J. Mukherjee, S.M. Petrinec and I.J. Sillanpaa, Journal of Geophysical Research: Space Physics 119, 2563 (2014).

[32] Y. Su, A.M. Veronig, G.D. Holman, B.R. Dennis, T. Wang, M. Temmer and W. Gan, Nat Phys 9, 489 (2013).

[33] E.G. Zweibel and M. Yamada, Annual Review of Astronomy and Astrophysics 47, 291 (2009).

[34] R. Schopper, H. Lesch and G.T. Birk, Astronomy and Astrophysics 335, 26 (1998).

[35] J. Burch, T.E. Moore, R. Torbert and B. Giles, Space Science Reviews, 1 (2015).

[36] J.A. Bittencourt, Fundamentals of Plasma Physics (Springer, Heidelberg, 2004), $3^{\text {rd }}$ ed.

[37] W. Baumjohann and R.A. Treumann, Basic Space Plasma Physics (Imperial College Press, London, 1997), $3^{\text {rd }}$ ed.

[38] D.P. Stern, Space Science Reviews 6, 147 (1966).

[39] P.A. Cassak, Catastrophe Model for the Onset of Fast Magnetic Reconnection. Tese de Doutorado, University of Maryland, 2006.

[40] J. Lemen, A. Title, D. Akin, P. Boerner, C. Chou, J. Drake, D. Duncan, C. Edwards, F. Friedlaender, G. Heyman, N. Hurlburt, N. Katz, G. Kushner, M. Levay, R. Lindgren, D. Mathur, E. McFeaters, S. Mitchell, R. Rehse, C. Schrijver, L. Springer, R. Stern, T. Tarbell, J.-P. Wuelser, C. Wolfson, C. Yanari, J. Bookbinder, P. Cheimets, D. Caldwell, E. Deluca, R. Gates, L. Golub, S. Park, W. Podgorski, R. Bush, P. Scherrer, M. Gummin, P. Smith, G. Auker, P. Jerram, P. Pool, R. Soufli, D. Windt, 
S. Beardsley, M. Clapp, J. Lang and N. Waltham, Solar Physics 275, 17 (2012).

[41] T.G. Forbes, Nat Phys 9, 456 (2013).

[42] W. Pesnell, B. Thompson and P. Chamberlin, Solar Physics 275, 3 (2012).

[43] P.A. Cassak, J.F. Drake and M.A. Shay, The Astrophysical Journal Letters 644, 145 (2006).

[44] W. Daughton, V. Roytershteyn, H. Karimabadi, L. Yin, B.J. Albright, B. Bergen and K.J. Bowers, Nat Phys 7, 539 (2011). 